JOURNAL OF

SYMPLECTIC GEOMETRY

Volume 3, Number 4, 531-543, 2005

\title{
TORIC SYMPLECTIC SINGULAR SPACES I: ISOLATED SINGULARITIES
}

\author{
Daniel M. Burns, Victor W. Guillemin, and Eugene M. Lerman
}

We generalize a theorem of Delzant classifying compact connected symplectic manifolds with completely integrable torus actions to certain singular symplectic spaces. The assumption on singularities is that if they are not finite quotient then they are isolated.

\section{Introduction}

It is an old question as to what the symplectic geometry analogue of a singular algebraic variety should be. For example, it is an exercise in Gromov's book [11, p. 343]. One would like to have a definition of a singular object in symplectic geometry (not to be confused with symplectic varieties in algebraic geometry) that includes, at the very least, symplectic quotients by proper Lie group actions and, perhaps, affine subvarieties of complex vector spaces $\mathbb{C}^{n}$. There are a number of reasons for wanting such a definition.

(1) Symplectic analogues of algebro-geometric objects shed new light on questions of symplectic and algebraic geometry, providing new understanding and new techniques. For instance, Alexeev and Brion [1] recently proved the existence of degenerations of spherical varieties to toric varieties. The proof is not geometric; it uses commutative algebra. It would be interesting to find a purely symplectic proof. And for that, one needs to understand what symplectic singular spaces are.

(2) An intrinsic definition of singular symplectic spaces will play a useful role in understanding the dynamics of symmetric Hamiltonian systems, in particular, in understanding the stability and bifurcation of relative equilibria and the equivariant analogue of the Liapunov-WeinsteinMoser centre theorem (see for example [10] and references therein).

(3) In the same spirit, a good definition of a singular symplectic space should allow one to develop an analogue of Floer homology and to 
start proving the existence of relative periodic orbits in symmetric Hamiltonian systems without resort to perturbative methods, that is, away from relative equilibria.

(4) Such a definition should also shed new light on quantization of singular systems.

What should a symplectic singular space be? Fourteen years ago, Sjamaar and Lerman proved [23] that symplectic quotients by actions of compact Lie groups are stratified spaces (the result is also true for proper actions of non-compact groups, see $[\mathbf{3}, \mathbf{1 0}])$. They also proved that symplectic quotients have a number of interesting properties, some of which were abstracted in a definition of a symplectic stratified space [23, Definition $1.12]$.

Definition 1.1. A symplectic stratified space is a stratified space $X$ together with a subalgebra $C^{\infty}(X)$ ("the algebra of smooth functions") of the algebra of continuous functions $C^{0}(X)$ such that

(1) each stratum $S$ of $X$ is a symplectic manifold,

(2) $C^{\infty}(X)$ is a Poisson algebra, and

(3) the embedding $S \hookrightarrow X$ is Poisson for each stratum $S$.

The last line means that for any function $f \in C^{\infty}(X)$, its restriction $\left.f\right|_{S}$ to any stratum $S$ is a smooth function in the ordinary sense, and for any two functions $f, g \in C^{\infty}(X)$, we have $\left\{\left.f\right|_{S},\left.g\right|_{S}\right\}_{S}=\left.\{f, g\}\right|_{S}$, where on the left $\{\cdot, \cdot\}_{S}$ denotes the Poisson bracket defined by the symplectic form on the stratum $S$. This tells us that a singular symplectic space should be a stratified space. Recall that a stratified space is a topological space with a locally finite decomposition into manifolds, which are called strata. Moreover, stratified spaces are defined recursively. Namely, a neighbourhood of a point is homeomorphic to a product of a ball with a cone on a compact stratified space, called the link of the singularity at the point. Points in the same stratum have isomorphic links.

The results of $[\mathbf{2 3}]$ also seem to suggest that a singular symplectic space should be some sort of a differential space in the sense of Aronszajn [2], Spallek [25] and Sikorski [22] (see [19] for the state of the art on differential spaces and a large bibliography). We feel, however, that this may not be the best way to approach symplectic singular spaces. For instance, consider a complex algebraic subvariety $X$ of $\mathbb{C}^{n}$. The most natural space of smooth functions on $X$ is the space of restrictions $\left.C^{\infty}\left(\mathbb{C}^{n}\right)\right|_{X}$, the space of Whitney smooth functions. But there is no obvious Poisson bracket in sight. Here is another example. Take $X=\mathbb{R}^{2}$ with the standard symplectic form and stratify it as $X=\left(\mathbb{R}^{2} \backslash\{0\}\right) \cup\{0\}$. Then for any $n$, the space of $\mathbb{Z} / n$-invariant functions on $\mathbb{R}^{2}$ is a Poisson algebra and embeddings of strata are Poisson. In this example, there are infinitely many choices of $C^{\infty}(X)$ and no preferred one. 
In this paper, we set ourselves a more modest goal. We investigate what a toric symplectic singular space (with isolated singularities) should be. The paradigm here is a theorem of Delzant [9]: compact symplectic manifolds with completely integrable torus actions are smooth projective toric varieties. The lesson here is that toric symmetries turn a floppy symplectic manifold into a rigid algebraic variety.

In the case of toric symplectic singular spaces, the links of singularities are toric contact spaces. As the classification of contact toric manifolds shows [14], in order for the moment map image to be locally convex, these contact toric spaces have to be of Reeb type. Recall that the notion of Reeb type was introduced by Boyer and Galicki in their study of Sasakian toric manifolds $[6]$.

Since symplectic orbifolds can be treated on the same footing as symplectic manifolds [16] and since the category of orbifolds is stable under symplectic cuts, we will assume that our spaces are stratified by orbifolds, not manifolds.

The main result of the paper is Theorem 1 . We prove that compact symplectic toric spaces, such that their non-orbifold singularities are isolated, are classified by labelled convex rational polytopes that are simple away from the vertices. Consequently they are isomorphic to symplectic quotients of $\mathbb{C}^{N}$ by actions of compact abelian Lie groups (i.e., products of tori $\mathbb{R}^{n} / \mathbb{Z}^{n}$ and of finite abelian groups). It then follows that all such spaces are projective toric varieties.

\section{Classification of toric symplectic spaces with isolated singularities}

In this section, we define symplectic toric stratified spaces with the property that their non-orbifold singularities are isolated ${ }^{1}$ and prove that they are classified by labelled rational polytopes that are simple away from vertices. We start with a few preliminary definitions and remarks.

One of the techniques of the proof involves blowing-up singularities. Now in the symplectic category blow-ups are symplectic cuts [12]. Symplectic cuts are symplectic quotients. So orbifold singularities are generic and thus unavoidable. On the other hand, the symplectic cuts of orbifolds are again, generically, orbifolds. So it is more convenient to work with orbifolds throughout. Therefore, we will consider stratified spaces stratified by orbifolds. ${ }^{2}$ Thus, for us, a singular space with isolated singularities

\footnotetext{
${ }^{1}$ Their orbifold singularities are arbitrary.

${ }^{2}$ There is a fair amount of confusion in the literature regarding the notions of orbifold and orbifold morphisms. In this paper we will only deal with reduced (effective) orbifolds, that is, orbifolds for which the structure groups all act effectively, as defined, for example, by Moerdijk and Pronk [18]. It seems to us that the correct notion of morphism of effective orbifolds is that of a strong map of Moerdijk and Prong (op. cit.). In many instances in symplectic geometry, the notion of a reduced orbifold is too restrictive. The main reason is
} 
is a Hausdorff topological space $M$ together with a discrete set of points $\left\{x_{\alpha}\right\} \subset M$ so that

$$
M_{\text {reg }}:=M \backslash\left\{x_{\alpha}\right\}
$$

is a smooth (meaning $C^{\infty}$ ) orbifold. We also want the neighbourhoods of singularities $x_{\alpha}$ to have bounded topology. Therefore, we require that for each index $\alpha$, there is a compact orbifold $L_{\alpha}$ (the link of the singularity at $x_{\alpha}$ ) so that a neighbourhood of $x_{\alpha}$ in $M$ is homeomorphic to the open cone

$$
\stackrel{\imath}{c}\left(L_{\alpha}\right):=\frac{\left([0, \infty) \times L_{\alpha}\right)}{\left(\{0\} \times L_{\alpha}\right)} .
$$

In fact, it will be more convenient for us to define the topological cone $\stackrel{c}{c}(L)$ on a space $L$ by

$$
\stackrel{c}{c}(L):=\frac{([-\infty, \infty) \times L)}{(\{-\infty\} \times L)}
$$

and to refer to the image $*$ of $(\{-\infty\} \times L)$ in $\stackrel{c}{c}(L)$ as the vertex of the cone. Here we think of $[-\infty, \infty)$ as the topological space which is homeomorphic to $[0, \infty)$ and contains $\mathbb{R}=(-\infty, \infty)$ as a dense open subset.

By a torus we mean a compact connected abelian Lie group. We denote the Lie algebra of a torus $G$ by $\mathfrak{g}$. We let $\mathfrak{g}^{*}=\operatorname{Hom}(\mathfrak{g}, \mathbb{R})$, the dual of the Lie algebra, and $\mathbb{Z}_{G}=\operatorname{ker}\{\exp : \mathfrak{g} \rightarrow G\}$, the integral lattice of $G$. A vector $v \in \mathbb{Z}_{G}$ is primitive if it is not a positive integer multiple of another vector in the lattice $\mathbb{Z}_{G}$. A codimension one face of a polytope is a facet. A polytope in $\mathfrak{g}^{*}$ is rational if the normals of all of its facets lie in the integral lattice $\mathbb{Z}_{G}$. In general, the normals to the facets of a polytope in $\mathfrak{g}^{*}$ lie in $\left(\mathfrak{g}^{*}\right)^{*}=\mathfrak{g}$. Our convention is that all the normals to the facets are primitive and outward pointing. A polytope in $\mathfrak{g}^{*}$ is simple if all the facets are in general position. In particular there are exactly dim $\mathfrak{g}^{*}$ edges coming out of every vertex. Thus a cube is simple while an octahedron is not. However, an octahedron is simple away from the vertices.

A symplectic toric $G$-orbifold is a triple $\left(M, \omega, \Phi: M \rightarrow \mathfrak{g}^{*}\right)$, where $M$ is a connected orbifold, $\omega$ is a symplectic form on $M$ and $\Phi$ is a moment map for an effective completely integrable action of a torus $G$ on $(M, \omega)$. We do not assume that our toric orbifolds are compact, nor do we assume that the moment maps are proper.

Remark 2.1. A classification theorem of Lerman and Tolman [16] asserts that compact connected effective symplectic toric $G$-orbifolds are classified by simple rational polytopes in $\mathfrak{g}^{*}$ together with a positive integer label attached to each facet. The need for the integer labels can be seen in the

that slice representations for locally free proper actions of Lie groups need not be effective. The general correct notion of orbifolds and their morphisms ("good maps") is probably the one due to Chen and Ruan [8]. It is a folklore wisdom that Chen and Ruan's definitions make orbifolds into stacks over the category of manifolds. 
following example. Consider the teardrop orbifold $X_{n}$, that is, consider an orbifold whose underlying topological space is homeomorphic to the two sphere and whose only singularity is modelled on $\mathbb{C} /(\mathbb{Z} / n)$. The orbifold carries a symplectic form which is invariant under a Hamiltonian action of the circle $S^{1}$. The associated polytope is a closed interval in $\mathfrak{g}^{*} \simeq \mathbb{R}$. The classification theorem in question tells us that the image of the singularity is one of the end points of the interval and that this facet of the interval should be labelled $n$. More generally it turns out that for symplectic toric orbifolds, the orbifold structure groups of points that the moment map sends to facets are cyclic. The integer labels are simply the orders of these groups.

For us, a symplectic cone is a symplectic orbifold $(N, \omega)$ together with a free proper action $\rho_{t}$ of the reals such that $\rho_{t}^{*} \omega=\mathrm{e}^{t} \omega$ for all $t \in \mathbb{R}$. Just as for manifolds, there is a one-to-one correspondence between symplectic cones and (co-oriented) contact orbifolds. If $\left(N, \omega, \rho_{t}\right)$ is a symplectic cone, then $L=N / \mathbb{R}$ is a contact orbifold. Conversely, if $L$ is a contact co-oriented orbifold, then its symplectization is a symplectic cone. These facts are standard for manifolds. Their proofs in the orbifold case carry over mutatis mutandis from the manifold case: replace every occurrence of the word "manifold" by "orbifold."

We will say that a symplectic cone $\left(N, \omega, \rho_{t}\right)$ is $G$-toric if there is an effective action of a torus $G$ on $N$, preserving $\omega$ and commuting with dilations $\rho_{t}$ and having the property that $2 \operatorname{dim} G=\operatorname{dim} N$. Now, whenever a group action on a symplectic cone preserves a symplectic form and commutes with dilations $\rho_{t}$, it is Hamiltonian since it preserves the contraction of $\omega$ with the vector field $\zeta$ generating the dilations $\rho_{t}$. Note that the associated moment map $\Phi$ defined by the primitive $\iota(\zeta) \omega$ is homogeneous: $\Phi\left(\rho_{t}(x)\right)=\mathrm{e}^{t} \Phi(x)$. Hence $\lim _{t \rightarrow-\infty} \Phi\left(\rho_{t}(x)\right)=0$, and $\Phi$ extends to a continuous map on the topological cone $\stackrel{c}{c}(N / \mathbb{R})=\{*\} \cup N$. Consequently, any moment map on a toric symplectic cone $N$ extends to a continuous map on the cone $\stackrel{c}{c}(N / \mathbb{R})$.

Definition 2.2. A symplectic toric cone $\left(N, \omega, \rho_{t}, \Phi: N \rightarrow \mathfrak{g}^{*}\right)$ is of Reeb type if there is a vector $X \in \mathfrak{g}$ so that $\langle\Phi(x), X\rangle>0$ for all $x \in N$. Here again $\Phi$ denotes a homogeneous moment map.

We will see below (Lemma 2.10) that if additionally the quotient $N / \mathbb{R}$ is compact and connected, then the moment map image $\Phi(N) \cup\{0\} \subset \mathfrak{g}^{*}$ is a cone on a simple rational polytope. If $N$ is a manifold, the converse is true as well: if $\Phi(N) \cup\{0\}$ is a cone on a simple rational polytope, then $\left(N, \omega, \rho_{t}, \Phi: N \rightarrow \mathfrak{g}^{*}\right)$ is of Reeb type. This follows from the classification of contact toric manifolds [14]. Note that, in general, even if $N / \mathbb{R}$ is compact, the cone $\Phi(N) \cup\{0\}$ need not be a convex cone. This happens, for example, when $N$ is the symplectization of an overtwisted 3-sphere [13].

We are now in a position to define the main object of this paper. 
Definition 2.3. A toric symplectic singular $G$-space with isolated singularities is a Hausdorff topological space $M$ with an effective action of a torus $G$, which has a number of properties. Namely, we assume the following.

(1) There is a discrete set of points $\left\{x_{\alpha}\right\} \subset M$ such that $M_{\text {reg }}:=M \backslash\left\{x_{\alpha}\right\}$ is a symplectic toric $G$-orbifold.

(2) Topologically the neighbourhoods of the singular points $x_{\alpha}$ are cones on compact orbifolds, which are not quotients of odd dimensional spheres by finite abelian groups. ${ }^{3}$

(3) Symplectically and equivariantly, the punctured neighbourhoods of singular points $x_{\alpha}$ are neighbourhoods of $-\infty$ in toric symplectic cones. Hence by the preceding remarks, the moment map $\Phi$ on $M_{\text {reg }}$ extends to a continuous function on $M$ which we will continue to denote by $\Phi$.

(4) For each singular point $x_{\alpha}$, there is a vector $Y_{\alpha} \in \mathfrak{g}$ so that $\langle\Phi-$ $\left.\Phi\left(x_{\alpha}\right), Y_{\alpha}\right\rangle>0$ on $U_{\alpha} \backslash x_{\alpha}$, where $U_{\alpha}$ is a $G$-invariant neighbourhood of $x_{\alpha}$. That is, the neighbourhoods of singularities are symplectic cones of Reeb type.

The condition (2) on the links insures that the singularities are genuine non-orbifold singularities.

Theorem 1. Let $\left(M, \omega, \Phi: M \rightarrow \mathfrak{g}^{*}\right)$ be a compact connected toric symplectic singular $G$-space with isolated singularities. Then,

(1) The moment image $\Delta:=\Phi(M) \subset \mathfrak{g}^{*}$ is a rational polytope, which is simple except possibly at vertices.

(2) The fibres of the moment map $\Phi: M \rightarrow \mathfrak{g}^{*}$ are $G$-orbits.

(3) The moment polytope $\Delta$ plus positive integers attached to the facets of $\Delta$ uniquely determine the topological space $M$, the singularities $\left\{x_{\alpha}\right\}$ and the symplectic toric $G$-orbifold $M_{\mathrm{reg}}=M \backslash\left\{x_{\alpha}\right\}$.

Remark 2.4. There is an easy converse to Theorem 1. Namely, given any rational polytope $\Delta \subset \mathfrak{g}^{*}$ which is simple away from vertices and a set of positive integers attached to facets, there exists a singular symplectic $G$-variety with moment image $\Delta$. The proof is essentially the same as in Delzant's original paper [9] modified as in [16] to take care of the positive integer labels attached to facets. We will say more about it later in the paper: see Theorem 2.11 and Remark 2.12.

Remark 2.5. In a series of papers, Prato $[\mathbf{2 0}, 21]$ and Battaglia and Prato $[\mathbf{4}, \mathbf{5}]$ extended Delzant's construction of symplectic toric manifolds from simple unimodular polytopes. Namely, they found a way to attach spaces to arbitrary polytopes. The spaces constructed by Battaglia and Prato are stratified by the so-called "symplectic quasifolds" (a quasifold is a space which is locally modeled by a vector space modulo the action of a discrete group; it is usually not Hausdorff).

\footnotetext{
${ }^{3}$ In other words, we want the singularities to be genuine non-orbifold singularities.
} 
We now proceed with the proof of Theorem 1 . We start by recalling what symplectic cuts are [12]. Let $(M, \omega)$ be a symplectic orbifold with an effective Hamiltonian action of a circle $T$ and an associated moment map $\mu: M \rightarrow \mathbb{R}$. Let $a$ be a regular value of $\mu$. Then the action of $T$ on the level set $\mu^{-1}(a)$ is locally free and

$$
M / /{ }_{a} T:=\frac{\mu^{-1}(a)}{T}
$$

is again naturally a symplectic orbifold by the reduction theorem of Marsden, Weinstein and Meyer. Consider now an orbifold with boundary

$$
\{\mu \geq a\}:=\{x \in M \mid \mu(x) \geq a\} .
$$

Define an equivalence relation $\sim$ on $\{\mu \geq a\}$ by identifying points on the boundary that lie on the same orbit of $T$. The following theorem is one of the main observations of $[\mathbf{1 2}]$.

Theorem 2.6. In the above notation, the topological space

$$
\bar{M}_{\geq a}:=\{\mu \geq a\} / \sim
$$

can be naturally given the structure of a symplectic orbifold so that the inclusion $M / / a T \hookrightarrow \bar{M}_{\geq a}$ is a symplectic embedding and the difference $\bar{M}_{\geq a} \backslash M / /{ }_{a} T$ is symplectomorphic to the open subset $\{\mu>a\}$ of $(M, \omega)$. Moreover the restriction $\left.\mu\right|_{\{\mu>a\}}$ descends to a moment map $\bar{\mu}: \bar{M}_{\geq a} \rightarrow \mathbb{R}$ for the action of $S^{1}$ on the cut space $\bar{M}_{\geq a}$.

Remark 2.7. Mutatis mutandis the statement holds also for

$$
\bar{M}_{\leq a}:=\{\mu \leq a\} / \sim .
$$

Remark 2.8. Theorem 2.6 is local: if $\Sigma \subset(M, \omega)$ is a hypersurface which separates $M$ into two manifolds with boundary $M^{+}$and $M^{-}$and if the null foliation of $\left.\omega\right|_{\Sigma}$ consists of closed leaves, then we may collapse the leaves of the null foliation in the boundaries of $M^{+}$and $M^{-}$and obtain symplectic orbifolds without boundary.

Remark 2.9. The results of Theorem 2.6 hold equivariantly. In particular, suppose a torus $G$ acts on $(M, \omega)$ with moment map $\Phi: M \rightarrow \mathfrak{g}^{*}$ and $T \subset G$ is a closed subgroup generated by $X \in \mathfrak{g}$ so that $\mu=\langle\Phi, X\rangle$. Then the cut space $\bar{M}_{\geq a}$ inherits a Hamiltonian action of $G$. Moreover the restriction $\left.\Phi\right|_{\{\mu>a\}}$ descends to a $G$-moment map $\bar{\Phi}$ on the cut space and

$$
\bar{\Phi}\left(\bar{M}_{\geq a}\right)=\Phi(\{\mu>a\})=\Phi(M) \cap\left\{\eta \in \mathfrak{g}^{*} \mid\langle\eta, X\rangle \geq a\right\} .
$$

Theorem 2.6 was motivated by symplectic blowups. Namely let

$$
(M, \omega)=\left(\mathbb{C}^{n}, \sqrt{-1} \sum d z_{j} \wedge \bar{z}_{j}\right)
$$


and let $\mu(z)=|z|^{2}$. Then $\bar{M}_{\geq a}$ is the symplectic blow-up of $\mathbb{C}^{n}$ at the origin and $\bar{M}_{\leq a}$ is $\mathbb{C} P^{n}$.

Next we sort out neighbourhoods of singular points.

Lemma 2.10. Let $\left(N, \omega, \rho_{t}\right)$ be a symplectic cone with an action of a torus $K$ which preserves the symplectic form and commutes with dilations $\rho_{t}$. Let $\mu: N \rightarrow \mathfrak{k}^{*}$ denote the associated homogeneous moment map. Suppose there is a vector $X \in \mathfrak{k}$ such that

$$
\langle\mu(x), X\rangle>0 \quad \text { for all } x \in N,
$$

that is, suppose $\left(N, \omega, \rho_{t}, \mu: N \rightarrow \mathfrak{k}^{*}\right)$ is of Reeb type. If the base $B=N / \mathbb{R}$ is compact and connected, then $\mu$ is proper as a map into $\mathfrak{k}^{*} \backslash\{0\}$, the fibers of $\mu$ are connected, and the moment cone $\mu(N) \cup\{0\}$ is a cone on a convex rational polytope.

Proof. Since the base $B$ of the cone is compact, (2.1) is an open condition. Therefore we may assume that $T=\{\exp t X \mid t \in \mathbb{R}\}$ is a circle. Then $f=\langle\mu, X\rangle$ is the moment map for the action of $T$ on $(N, \omega)$. Recall that $\mu$ is defined by

$$
\langle\mu, Y\rangle=\omega\left(\zeta, Y_{N}\right)
$$

for all $Y \in \mathfrak{k}$, where $\zeta$ is the vector field generating $\rho_{t}$ and $Y_{N}$ denotes the vector field on $N$ induced by $Y$. Consequently $\mu\left(\rho_{t}(x)\right)=\mathrm{e}^{t} \mu(x)$ for all $t \in \mathbb{R}, x \in M$. Note also that since $f=\omega\left(\zeta, X_{N}\right)>0$ by assumption, $X_{N} \neq 0$ anywhere on $N$; hence the action of $T$ on $N$ is locally free.

We now argue that $f^{-1}(1)$ is diffeomorphic to $B$, hence is compact and connected. Since

$$
f\left(\rho_{t}(x)\right)=\mathrm{e}^{t} f(x)
$$

the $\rho_{t}$-orbit map $\pi: f^{-1}(1) \rightarrow B$ is injective. Moreover, it is onto: pick a section $s: B \rightarrow N$ of $\pi: N \rightarrow B$ (any principal $\mathbb{R}$-bundle is trivial so such a global section does exist). Let

$$
\tau(x)=-\log (f(s(x))) .
$$

Then

$$
f\left(\rho_{\tau(x)}(s(x))\right)=\mathrm{e}^{\tau(x)} f(s(x))=1 .
$$

Note that $f^{-1}(1) \hookrightarrow N$ is a $K$-invariant section for $\pi: N \rightarrow B$. Hence $N$ is $K$-equivariantly diffeomorphic to $B \times \mathbb{R}$. Moreover under this identification of $N$ with $B \times \mathbb{R}, f$ is simply the map $f(m, t)=\mathrm{e}^{t}$. Therefore, $f$ is proper as a map into $(0, \infty)$. Hence $\mu: N \rightarrow\left\{\eta \in \mathfrak{k}^{*} \mid\langle\eta, X\rangle>0\right\}$ is proper as well. By [15, Theorem 4.3], $\mu(N)$ is convex and the fibres of $\mu$ are connected. But more can be said.

The restriction $\left.\mu\right|_{f^{-1}(1)}$ descends to a moment map $\bar{\mu}$ for the action of $K$ on the quotient $f^{-1}(1) / T$, which is a compact symplectic orbifold. By the orbifold version of the Atiyah-Guillemin-Sternberg convexity theorem [16] $\bar{\mu}\left(f^{-1}(1) / T\right)$ is a rational polytope. Hence $\mu\left(f^{-1}(1)\right)=\bar{\mu}\left(f^{-1}(1) / T\right)$ is a 
rational polytope as well. Therefore, $\mu(N) \cup\{0\}=\mathbb{R}^{>0} \mu\left(f^{-1}(1)\right) \cup\{0\}$ is a cone on a rational polytope.

Proof of Theorem 1. Since $M$ is compact, the set of singularities $\left\{x_{\alpha}\right\}$ is finite. By assumption, a neighbourhood $U_{i}$ of each singular point $x_{i}$ is the neighbourhood of $-\infty$ in a toric symplectic cone of Reeb type. In particular, there is a vector $Y_{i}$ in the integral lattice $\mathbb{Z}_{G}$ of $G$ such that $\left\langle\Phi-\Phi\left(x_{i}\right), Y_{i}\right\rangle>0$ on $U_{i}$. By Lemma 2.10, the restriction of the function $f_{i}=\left\langle\Phi, Y_{i}\right\rangle$ to $U_{i}$ is proper as a map onto its image. By the proof of the lemma, the action of the circle $G_{i}:=\left\{\exp t Y_{i} \mid t \in \mathbb{R}\right\}$ is locally free on $U_{i} \backslash\left\{x_{i}\right\}$.

Now for every sufficiently small $\varepsilon>0$ consider

$$
M(\varepsilon):=M \backslash \bigcup_{i}\left\{m \in U_{i} \mid f_{i}(m)<f_{i}\left(x_{i}\right)+\varepsilon\right\} ;
$$

it is a compact symplectic orbifold with boundary. The connected components of the boundary are the contact toric orbifolds $\left\{m \in U_{i} \mid f_{i}(m)=\right.$ $\left.\varepsilon+f_{i}\left(x_{i}\right)\right\}$. Moreover by Theorem 2.6 and subsequent remarks, if we divide each component by the corresponding group $G_{i}$, the various components of the boundary disappear and the result is a compact symplectic toric orbifold $\bar{M}(\varepsilon)$. Furthermore, $\left.\Phi\right|_{M(\varepsilon)}$ descends to a moment map $\bar{\Phi}$ on $\bar{M}(\varepsilon)$ for the induced action of $G$. By construction, $\Phi(M(\varepsilon))=\bar{\Phi}(\bar{M}(\varepsilon))$ and the fibres of $\left.\Phi\right|_{M(\varepsilon)}$ are $G$-orbits if and only if the fibres of $\bar{\Phi}$ are. Since $\bar{M}(\varepsilon)$ is a symplectic toric orbifold, $\bar{\Phi}(\bar{M}(\varepsilon))$ is a simple rational polytope [16]. Moreover, the facets of $\bar{\Phi}(\bar{M}(\varepsilon))$ that come from cuts have labels 1 attached to them since cutting does not introduce any spurious orbifold singularities (Remark 2.1). Therefore, $\Phi(M(\varepsilon))$ is a simple rational polytope and the fibres of $\left.\Phi\right|_{M(\varepsilon)}$ are $G$-orbits. Note that

$$
\bigcup_{\{\varepsilon>0\}} M(\varepsilon)=M_{\mathrm{reg}} .
$$

We are now in a position to argue that $\Phi(M)$ is convex and that the fibres of $\Phi: M \rightarrow \mathfrak{g}^{*}$ are $G$-orbits. Suppose $a, b \in M_{\text {reg }}$ are two points. Pick $\varepsilon$ small enough so that $a, b \in M(\varepsilon)$. Since $\Phi(M(\varepsilon)) \subset \Phi\left(M_{\text {reg }}\right)$ is convex, the line segment $[\Phi(a), \Phi(b)]$ is entirely contained in $\Phi(M(\varepsilon))$, hence in $\Phi\left(M_{\text {reg }}\right) \supset \Phi(M(\varepsilon))$. Therefore, $\Phi\left(M_{\text {reg }}\right)$ is convex. Also, if $a, b \in M_{\text {reg }}$ and $\Phi(a)=\Phi(b)$, then we can choose $\varepsilon$ so that $a, b \in M(\varepsilon)$. Hence $a \in G \cdot b$. Therefore, the fibres of $\Phi: M \rightarrow \mathfrak{g}^{*}$ are $G$-orbits. We will need this fact in the proof of uniqueness below.

Since $M_{\text {reg }}$ is dense in $M, \Phi(M)$ is contained in the closure $\overline{\Phi\left(M_{\text {reg }}\right)}$. On the other hand, since $M$ is compact, $\Phi(M)$ is closed. Hence $\Phi(M)=$ $\overline{\Phi\left(M_{\mathrm{reg}}\right)}$. In particular, $\Phi(M)$ is convex.

We now argue that $\Phi(M)$ is a polytope, that the points $\Phi\left(x_{i}\right)$ are among the vertices of this polytope and that $\Phi^{-1}\left(\Phi\left(x_{i}\right)\right)=\left\{x_{i}\right\}$ for all $i$. We cut 
off the conical neighbourhoods of singularities. That is, for sufficiently small $\varepsilon$, we have

$$
M=M(\varepsilon) \cup \bigsqcup_{i}\left\{m \in U_{i} \mid f_{i}(m) \leq f_{i}\left(x_{i}\right)+\varepsilon\right\}
$$

We know that both $\Phi(M(\varepsilon))$ and

$$
\Delta_{i}(\varepsilon):=\Phi\left(\left\{f_{i} \leq f_{i}\left(x_{i}\right)+\varepsilon\right\}\right)
$$

are convex polytopes which have disjoint interiors (the latter is true because the fibres of $\Phi: M_{\text {reg }} \rightarrow \mathfrak{g}^{*}$ are $G$-orbits). Also, for each $i, \Delta_{i}(\varepsilon)$ intersects $\Phi(M(\varepsilon))$ in a facet that does not contain the vertex $\Phi\left(x_{i}\right)$. Hence $\Phi(M)$ is a polytope, $\Phi\left(x_{i}\right)$ are vertices (not necessarily all the vertices) and, since $\left.\Phi\right|_{\left\{m \in U_{i} \mid f_{i}(m) \leq f_{i}\left(x_{i}\right)+\varepsilon\right\}}$ has the property that the fibre above $\Phi\left(x_{i}\right)$ is the point $x_{i}$, we have that the preimages of vertices of $\Phi(M)$ are single points. We leave it to the reader to check that $\Phi(M)$ is simple except possibly at the vertices.

Uniqueness. Suppose $\left(M_{i}, \omega_{i}, \Phi_{i}: M_{i} \rightarrow \mathfrak{g}^{*},\left\{x_{1}^{(i)}, \ldots, x_{N}^{(i)}\right\}\right), i=1,2$, are two toric symplectic singular $G$-spaces. Suppose $\Phi_{1}\left(M_{1}\right)=\Phi_{2}\left(M_{2}\right)$ and the associated integer labels on the facets agree. Since by assumption the links of singularities are not finite quotients of odd dimensional spheres, a vertex of the polytope $\Phi_{i}\left(M_{i}\right)$ is simple if and only if it is the image of a point in $\left(M_{i}\right)_{\text {reg. }}$. Therefore, after some renumbering, $\Phi_{1}\left(x_{i}^{(1)}\right)=\Phi_{2}\left(x_{i}^{(2)}\right)$.

The symplectic toric orbifolds $\left(\left(M_{i}\right)_{\mathrm{reg}}, \omega_{i}, \Phi_{i}\right), i=1,2$, are locally isomorphic in the sense of $[\mathbf{1 6}, \mathrm{p} .4222]$. That is, every point $p \in \Phi_{1}\left(\left(M_{1}\right)_{\mathrm{reg}}\right)=$ $\Phi_{2}\left(\left(M_{2}\right)_{\text {reg }}\right)$ has an open neighbourhood $U \subset \Delta_{\text {reg }}$ and an equivariant diffeomorphism $\phi_{U}: \Phi_{1}^{-1}(U) \rightarrow \Phi_{2}^{-1}(U)$ with $\phi_{U}^{*} \omega_{2}=\omega_{1}$ and $\phi_{U}^{*} \Phi_{2}=\Phi_{1}$ on $\Phi_{1}^{-1}(U)$. Hence, to prove uniqueness, it is enough to argue that the group $H^{2}\left(\Phi_{i}\left(\left(M_{i}\right)_{\mathrm{reg}}, \mathbb{Z}_{G}\right)\right.$ is trivial [16, Section 7]. But $\Phi_{i}\left(\left(M_{i}\right)_{\mathrm{reg}}\right)$ is a convex polytope with some vertices possibly deleted, hence is contractible. Thus the uniqueness follows and we get an equivariant diffeomorphism $\phi:\left(M_{1}\right)_{\text {reg }} \rightarrow\left(M_{2}\right)_{\text {reg }}$ with $\phi^{*} \omega_{2}=\omega_{1}$ and $\phi^{*} \Phi_{2}=\Phi_{1}$. It remains to argue that $\phi$ extends to a continuous map $\phi: M_{1} \rightarrow M_{2}$ so that $\phi^{*} \Phi_{2}=\Phi_{1}$ still holds.

Fix $i$. Take any sequence $\left\{y_{n}\right\}$ in $\left(M_{1}\right)_{\text {reg }}$ converging to $x_{i}^{(1)}$. Then,

$$
\lim _{n \rightarrow \infty} \Phi_{2}\left(\phi\left(y_{n}\right)\right)=\lim _{n \rightarrow \infty}\left(\Phi_{1}\left(y_{n}\right)\right)=\Phi_{1}\left(x_{i}^{(1)}\right)=\Phi_{2}\left(x_{i}^{(2)}\right) .
$$

Since $M_{2}$ is compact, we may assume that $\left\{\phi\left(y_{n}\right)\right\}$ converges. Since $\Phi_{2}\left(\lim _{n \rightarrow \infty} \phi\left(y_{n}\right)\right)=\Phi_{2}\left(x_{i}^{(2)}\right)$, and since $\Phi_{2}^{-1}\left(\Phi_{2}\left(x_{i}^{(2)}\right)\right)=\left\{x_{i}^{(2)}\right\}, \lim _{n \rightarrow \infty}$ $\phi\left(y_{n}\right)=x_{i}^{(2)}$. Since the sequence $\left\{y_{n}\right\}$ is arbitrary, $\phi$ does extend to a continuous map $\phi: M_{1} \rightarrow M_{2}$.

Theorem 1 has an easy converse which is a slight generalization of $[\mathbf{1 6}$, Theorem 8.2]. Namely, 
Theorem 2.11. Let $G$ be a torus. Let $\mathfrak{g}$ denote its Lie algebra, and let $\mathbb{Z}_{G} \subset \mathfrak{g}$ denote its integral lattice. Given a rational polytope $\Delta \subset \mathfrak{g}^{*}$, which is simple except possibly at the vertices, and a positive integer $m_{F}^{\circ}$ attached to each open facet $\stackrel{\circ}{F}$ of $\Delta$, there exists a compact toric symplectic singular $G$-space $\left(M, \omega, \Phi: M \rightarrow \mathfrak{g}^{*},\left\{x_{\alpha}\right\}\right)$ such that $\Phi(M)=\Delta$ and the orbifold structure group of a point in $M$ which maps to a open facet $\stackrel{\circ}{F}$ is $\mathbb{Z} / m_{F} \mathbb{Z}$.

Moreover, $\left(M, \omega, \Phi: M \rightarrow \mathfrak{g}^{*},\left\{x_{\alpha}\right\}\right)$ is a symplectic quotient of $\mathbb{C}^{N}$ by a closed abelian subgroup of $S U(N)$, where $N$ is the number of facets of $\Delta$ minus $\operatorname{dim} G$.

The proof is a straightforward modification of the proof of $[\mathbf{1 6}$, Theorem 8.2]. We omit it.

Remark 2.12. Combining the above theorem with the uniqueness part of Theorem 1, we see that every toric symplectic singular space is a symplectic quotient of $\mathbb{C}^{N}$. Therefore, by [24, Theorem 2.8], every toric symplectic singular space is a complex analytic space. Furthermore by [24, Lemma 2.16] it is a Kähler space in the sense of Grauert. The Kähler structures on these spaces are described elsewhere [7].

Remark 2.13. We close with the most elementary example of a space of the kind described in the main theorem. Since the main theorem is a uniqueness theorem, we describe the example as a toric variety first and then describe its symplectic structure and its moment polytope. Consider the singular quadric hypersurface $V=\left\{\left[\zeta_{0}: \ldots: \zeta_{4}\right] \in \mathbb{C P}^{4} \mid \zeta_{1} \zeta_{2}+\zeta_{3} \zeta_{4}=0\right\}$, with an isolated singularity at $[1: 0: 0: 0: 0] .(\lambda, \mu, \nu) \in\left(\mathbb{C}^{*}\right)^{3}$ acts on $V$ by

$$
(\lambda, \mu, \nu) \cdot\left[\zeta_{0}: \cdots: \zeta_{4}\right]=\left[\zeta_{0}: \lambda \zeta_{1}: \mu \zeta_{2}: \nu \zeta_{3}: \lambda \mu \nu^{-1} \zeta_{4}\right] \text {. }
$$

Note that $\left(\mathbb{C}^{*}\right)^{3}$ has four fixed points $([0: 1: 0: 0: 0]$, and permutations) in the hyperplane $\zeta_{0}=0$ in addition to the singular point being fixed. $\left(\mathbb{C}^{*}\right)^{2} \cong\left(\mathbb{C}^{*}\right)^{3} /\{\lambda=\mu=\nu\}$ acts on $V \cap\left\{\zeta_{0}=0\right\}$. For any compatible symplectic form on $V$, the moment map image for this action will be the convex hull of the images of the five fixed points which generate a "pyramid," the simplicial cone over the quadrilateral coming from the moment map of $\left(\mathbb{C}^{*}\right)^{2}$ on $V \cap\left\{\zeta_{0}=0\right\}$. The one singular vertex, the vertex of the pyramid, has four edges emanating from it, corresponding to the embedding dimension of the singular point of $V$. To be specific, to verify the homogeneity for the restriction of the Fubini-Study form on $\mathbb{C P}^{4}$ to $V$ near the singular point, consider the affine space $\mathbb{C}^{4}$ with coordinates $z_{i}=\zeta_{i} / \zeta_{0}, i=1, \ldots, 4 . V \cap \mathbb{C}^{4}=\left\{z_{1} z_{2}+z_{3} z_{4}=0\right\}$, and $\omega=\frac{i}{2} \partial \bar{\partial} \log \left(\frac{1}{1+\|z\|^{2}}\right)$, where $\|z\|^{2}=\sum\left|z_{i}\right|^{2}$, restricted to $V$. The infinitesimal generator of the dilations $\rho_{t}$ in the definition of symplectic cone above is given by $\xi=\frac{1}{2}\left(1+r^{2}\right) r \partial / \partial r$. The vertices of the polytope for this symplectic form are $(0,0,0),(1,0,0),(0,1,0),(0,0,1)$ and $(1,1,-1)$. There are 
no orbifold points in this example, so there are no integer labels (not equal to 1) on the polytope facets.

\section{References}

[1] V. Alexeev and M. Brion, Toric degenerations of spherical varieties, Selecta Math. (NS) 10 (2004), 453-478.

[2] N. Aronszajn, Subcartesian and subriemannian spaces, Not. Am. Math. Soc. 14 (1967), 111.

[3] L. Bates and E. Lerman, Proper group actions and symplectic stratified spaces, Pac. J. Math. 181(2) (1997), 201-229.

[4] F. Battaglia and E. Prato, Generalized toric varieties for simple nonrational convex polytopes, Internat. Math. Res. Notices (2001), no. 24, 1315-1337.

[5] F. Battaglia and E. Prato, Nonrational, nonsimple convex polytopes in symplectic geometry, Electron. Res. Announc. Am. Math. Soc. 8 (2002), 29-34 (electronic).

[6] C. Boyer and K. Galicki, A note on toric contact geometry, J. Geom. Phys. 35(4) (2000), 288-298.

[7] D. Burns, V. Guillemin and E. Lerman, Kähler metrics on singular toric varieties, arXiv: math.DG/0501311.

[8] W. Chen and Y. Ruan, Orbifold Gromov-Witten theory, in 'Orbifolds in mathematics and physics (Madison WI, 2001)', 25-85, Contemp. Math., 310, Amer. Math. Soc., Providence, RI, 2002.

[9] T. Delzant, Hamiltoniens périodiques et images convexes de l'application moment, Bull. Soc. Math. France 116(3) (1988), 315-339.

[10] V. Ginzburg and E. Lerman, Existence of relative periodic orbits near relative equilibria, Math. Res. Lett. 11(2, 3) (2004), 397-412.

[11] M. Gromov, Partial differential relations, Ergebnisse der Mathematik und ihrer Grenzgebiete (3), 9, Springer-Verlag, Berlin, 1986, x + 363 pp. ISBN: 3-540-12177-3.

[12] E. Lerman, Symplectic cuts, Math. Res. Lett. 2(3) (1995), 247-258.

[13] E. Lerman, Contact cuts, Israel J. Math. 124 (2001), 77-92.

[14] E. Lerman, Contact toric manifolds, J. Symplectic Geom. 1(4) (2003), 785-828.

[15] E. Lerman, E. Meinrenken, S. Tolman and C. Woodward, Nonabelian convexity by symplectic cuts, Topology 37(2) (1998), 245-259.

[16] E. Lerman and S. Tolman, Symplectic toric orbifolds, Trans. A.M.S. 349 (1997), 4201-4230.

[17] E. Lerman and C. Willett, The topological structure of contact and symplectic quotients, Internat. Math. Res. Notices (2001), no. 1, 33-52.

[18] I. Moerdijk and D.A. Pronk, Orbifolds, sheaves and groupoids K-Theory 12(1) (1997), $3-21$.

[19] J.A. Navarro González and J.B. Sancho de Salas, $C^{\infty}$-differentiable spaces, Lecture Notes Math., 1824, Springer-Verlag, Berlin, 2003, ISBN 3-540-20072-X.

[20] E. Prato, Sur une généralisation de la notion de V-variété, C. R. Acad. Sci. Paris Sr. I Math. 328(10) (1999), 887-890.

[21] E. Prato, Simple non-rational convex polytopes via symplectic geometry, Topology 40(5) (2001), 961-975. 
[22] R. Sikorski, Abstract covariant derivative, Colloq. Math. 18 (1967), 251-272.

[23] R. Sjamaar and E. Lerman, Stratified symplectic spaces and reduction, Ann. Math. 134 (1991), 375-422.

[24] R. Sjamaar, Holomorphic slices, symplectic reduction and multiplicities of representations, Ann. Math. 141 (1995), 87-129.

[25] K. Spallek, Differenzierbare Räume, Math. Ann. 180 (1969), 269-296.

UNIVERSITY OF MiChIGAN

Ann Arbor, MI 48109

E-mail address: dburns@umich.edu

M.I.T.

CAmbridge, MA 02139

E-mail address: vwg@math.mit.edu

UNIVERSITY OF ILLINOIS

URBANA, IL 61801

Australian National University

Canberra ACT 0200, Australia

E-mail address: lerman@math.uiuc.edu

Received 01/20/2005, accepted 06/23/2005. Supported in part by NSF grants DMS0104047 (DB), DMS-0104116 (VG) and DMS-0204448(EL). 
